\title{
Magnetic instability of filaments in different solar regions
}

\author{
J. Palacios, A. Guerrero, C. Cid, E. Saiz and Y. Cerrato \\ Departamento de Física y Matemáticas, \\ Universidad de Alcalá \\ University Campus, Sciences Building, P.O. 28871, Alcalá de Henares, Spain \\ email: judith.palacios@uah.es
}

\begin{abstract}
Magnetic instability is a key consideration for filament eruptions and subsequent CMEs. In this contribution we are considering different magnetic conditions for active and nonactive regions, such as coronal hole regions and quiet sun, and also active regions of a simple magnetic configuration. The aim is to assess magnetic instability through potential and nonpotential field modelling and 3D evaluation of the magnetic decay index. Some eruptive examples from solar cycle 24 using $\mathrm{HMI} / \mathrm{SDO}$ data are presented, complemented with observations of $\mathrm{AIA} / \mathrm{SDO}$.
\end{abstract}

Keywords. Sun: magnetic fields, Sun: atmosphere

\section{Introduction}

Solar magnetic fields are essential for understanding solar activity and its evolution along the solar cycle, since formation of active regions, and other critical structures as filaments - and open field areas as coronal holes - depend on this evolution.

Filament eruption is a dominant process which, when it succeeds, usually leads to a coronal mass ejection (CME). These phenomena are a primary interest for space weather purposes. At the Spanish Space Weather Service (http: //www . senmes . es/index-en.php) we aim at reconstructing the Sun-to-Earth chain, which appears in our daily reports and it is the basis for forecasting geomagnetic storms.

Some features of interest are actually not the most violent (nor extremely conspicuous) CMEs, but the stealth and very faint CMEs, that also can induce geomagnetic storms. A first example case is shown by Palacios et al.(2015), as an event of a smooth filament eruption that led to a moderate geomagnetic storm at ground. This event consisted of a filament that peeled off very slowly, and the actual eruption may be helped by a supergranular-size flux emergence event underneath. The solar region where it was located and the helicity was the appropriate for triggering a geomagnetic storm. Another interesting case due to its stealthness was the eruption in the rim of a large coronal hole that provoked a faint CME and a moderate geomagnetic storm (Cid et al. 2016).

A fundamental physical fact for a filament eruption is instability. Filaments may be formed by different processes (see e.g. Mackay et al. 2010), and at some point in the evolution they may erupt, gradually or in a more violent way. There is a number of physical mechanisms about magnetic instabilities that undergoes a filament eruption: there are subsurface and surface mechanisms such as diffusion of magnetic flux and twisting (Amari et al. 2003b); slowly converging magnetic elements (Amari et al. 2003a); newly emerging flux (Feynman \& Martin 1995); flux cancellation and shearing (van Ballegooijen \& Martens 1989); simulated active region rotations and flux dispersal, as in Aulanier et al.(2010) or very recently in Zuccarello et al.(2015). Most of these mechanisms, apply 
not only for destabilizing but also for flux rope formation, producing shear, twist and flux storage in the rope; they are storage-and-release models. Other kind of mechanisms are the ideal MHD instabilities, as kink instabilities (e.g. Török \& Kliem 2005) or catastrophic loss of equilibrium (Forbes \& Priest(1995) and Forbes \& Lin(2000), references therein). The breakout model (Antiochos et al. 1999), as reconnection between a rising flux rope and the coronal arcade) and tether cutting (Moore et al.(2001), implying reconnection below) are relevant eruption models. Some of these models consider not only flux storage but also the magnetic field weakening of the overlying arcades.

Torus instability (Bateman 1978) is an ideal MHD instability (and a loss-of-equilibrium-like instability) consisting in the decay with height of the strapping (toroidal) magnetic field in comparison with the Lorentz self-force (Kliem \& Török(2006), Török \& Kliem(2007)). When the second dominates, a theoretical flux rope, assumed as a wire, may be unstable when expanding.

Aulanier(2014) classifies this amount in mainly two: breakout-like and torus-like instability. These two classify as models with decreasing magnetic tension.

A way of estimating and expressing this magnetic instability is the magnetic decay index $n$. This is computed as:

$$
n=-R \frac{\partial \ln \left(B_{e x}\right)}{\partial R}
$$

The index range for assessing instabilities is a critical value of about $n \approx 1.5$, depending how the magnetic field decreases with height. The actual figure depends on the geometry of the theoretical current wire model, being $n=1$ for straight wires to $n=1.5$ for a curved (semicircumference-shaped) wire of radius $R$. Filippov et al.(2014) suggest values of $n$ close to 0 close to the photosphere, up to $n=3$ for dipolar far field. However, recent estimations on theoretical simulations show that this is around $\mathrm{n} \approx 1.4-1.5$ with a difference of $7 \%$ in numerical experiments (Zuccarello et al.(2015)), about of 1.5-1.8 (Zuccarello et al.(2016)); or even larger, depending on the considerations in the simulations (references therein Zuccarello et al.(2015)).

This index is convenient to classify eruptions in gradual or more impulsive and associate it with the subsequent CME speed, as in Xu et al.(2012) and Török \& Kliem(2007). An interesting case of a filament eruption in a flaring active region is presented in Zuccarello et al.(2014).

\section{Data and cases}

Data for estimating the decay index $n$ were two types: quasi-synoptic data from HMI/SDO (Scherrer et al. (2012)) as input to compute the potential field source surface (PFSS) extrapolation (Schrijver \& De Rosa(2003)), extrapolated from $1 \mathrm{R}_{s}$ to $2 \mathrm{R}_{s}$. The other type of data used are the inversion products on SHARPS/HMI (Hoeksema et al.(2014)) for computing non-linear force-free field (NLFFF) extrapolation (Wheatland et al.(2000)), in the potential component for comparison. $B_{e x}$ is considered here as the non-radial magnetic field in the extrapolation. The heights of the NLFFF are variable depending on cases but less than $1.2 R_{s}$.

\subsection{September 2013}

The decay index was estimated in Palacios et al.(2015) for the case of a filament smooth eruption. A large filament peeled off from the chromosphere during one hour. This case has the particularity of exhibiting an important flux emergence event below the filament 


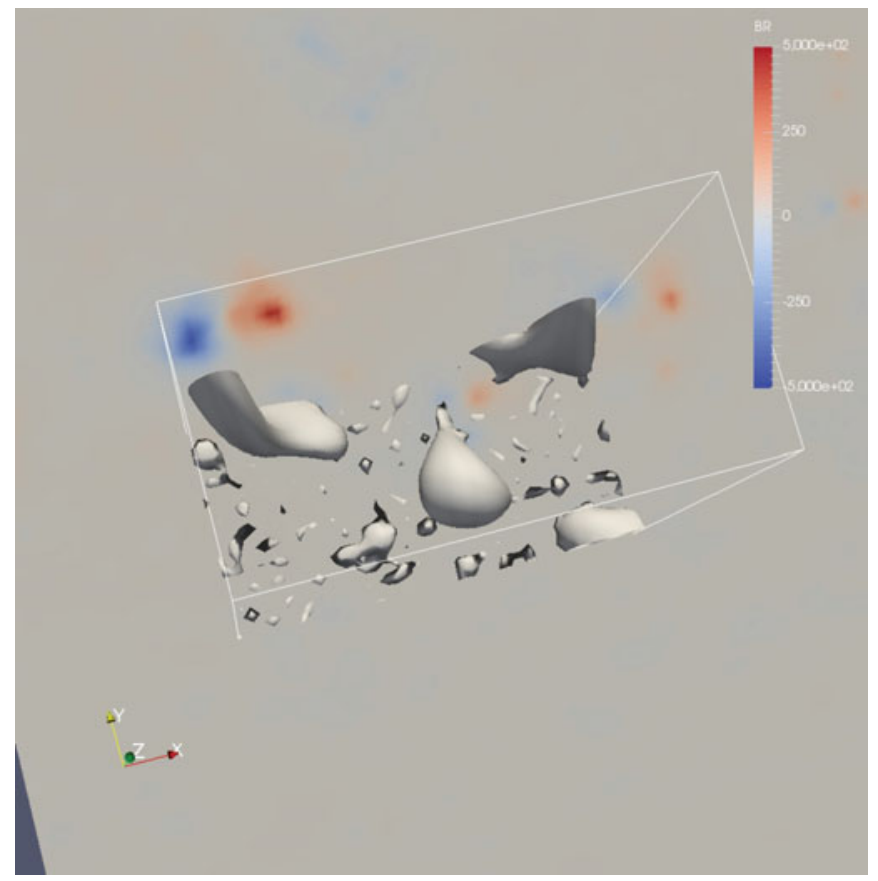

Figure 1. Decay index in the region of interest on 2 January 2015.

and related to a barb. In that work the decay index proved useful to trying to assess the instability in the filament that softly erupted. As suggested by Török \& Kliem(2007), a moderate $n$ and large $R_{o}$ (wire radius or equivalently, the apex height over the photosphere) can help on eruptions. The CME originated by this eruption and then the subsequent interaction with the Earth's magnetosphere led to a moderate geomagnetic storm at ground.

\subsection{January 2015}

The second case features the eruption of a quasi-stealth CME that drove a moderate (almost intense) geomagnetic storm at ground, in combination with the fast solar wind of a large coronal hole. The solar and interplanetary features are described by Cid et al. 2016 and also elsewhere in this Proceedings Volume.

Since the eruption area was located in the coronal hole boundary close to the southern solar pole, there are not SHARPS data because they are intended to include only active regions, so the heliographic location of SHARPS/HMI are always in the activity belts. Therefore the computation with SHARPS/HMI and NLFFF could not be performed. Instead, HMI quasi-synoptic data and PFSS extrapolation was used to compute the decay index for the 2 January 201512 UT, although the eruption happened during late January 2 up to several hours of the next day. The magnetic configuration of the region of interest is simple, a polarity inversion line (PIL) close to the area of unipolar field. The CME exhibited coronal eruption but not a chromospheric counterpart.

The computation of the decay index is shown in Figure 1. The critical decay index $n_{c}=1.5$ is shown as white surfaces, while darkest hues (red and blue in the color version) mark the areas of largest values of the radial magnetic field as a reference, in the figure legend. The upper-right surface (close to AR 12253) and bulb-shaped-center surface may 


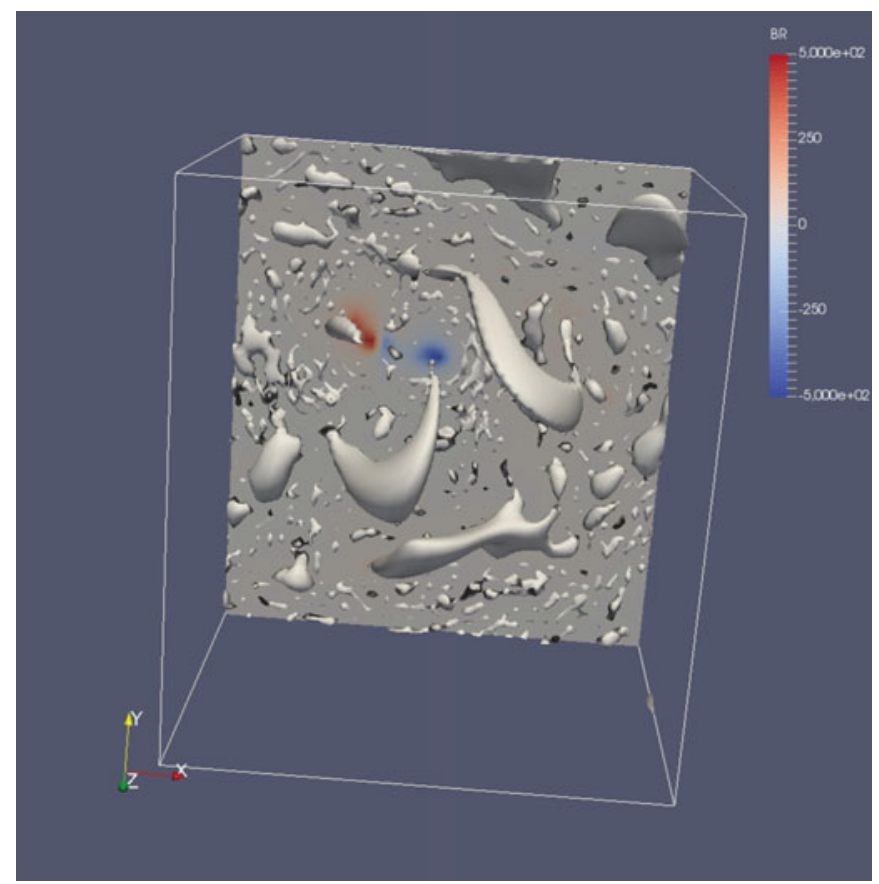

Figure 2. Decay index estimated by PFSS extrapolation in 21 June 2015.

correspond to a filament volume and the place where the CME left a dimming (just south of AR 12252), respectively.

\subsection{June 2015}

This is a very different case, since there were several CMEs produced in or close to the AR12371 that led to one of the most intense geomagnetic storms of the solar cycle 24, as registered by different geomagnetic indices on June 23 (Dst peak <-200 nT). The solar source is reported and geomagnetic storm is described by Palacios et al.(2016). The active region is a $\beta \gamma \delta$ anemone region. Two very fast CMEs were ejected $\left(>1000 \mathrm{~km} \mathrm{~s}^{-1}\right)$ on June 21 and 22, respectively. On June 23 there was an important filament eruption at the edge of this region, giving place to a CME of about $900 \mathrm{~km} \mathrm{~s}^{-1}$. These high speeds of a CME are a particularity, since there are not that many high-speed CMEs in the solar cycle 24. In addition to this, there were fast halo CMEs on June 18 and 19, with the combination of fast solar wind from a coronal hole.

In Figure 2 the white surfaces are shown where the critical decay index is equal to 1.5, and the darkest areas (or most intense coloured) highlight the largest values of the radial magnetic field. The half-moon shaped surfaces may correspond to the volume of an active filament and a quiescent filament, erupting on June 21 and 23, respectively.

In Figure 3 top panel, there is an AIA/HMI composite showing the complexity of the AR 12371 in the left panel, depicting the photosphere and corona in the CME on June 21. In the bottom panel, the critical decay index as surface. It has been computed through NLFFF with SHARPS/HMI data. The large half-moon surfaces do not appear since the volume is much more reduced in all the dimensions, enclosing only the AR and not the large PILs that are present in Figure 2. 


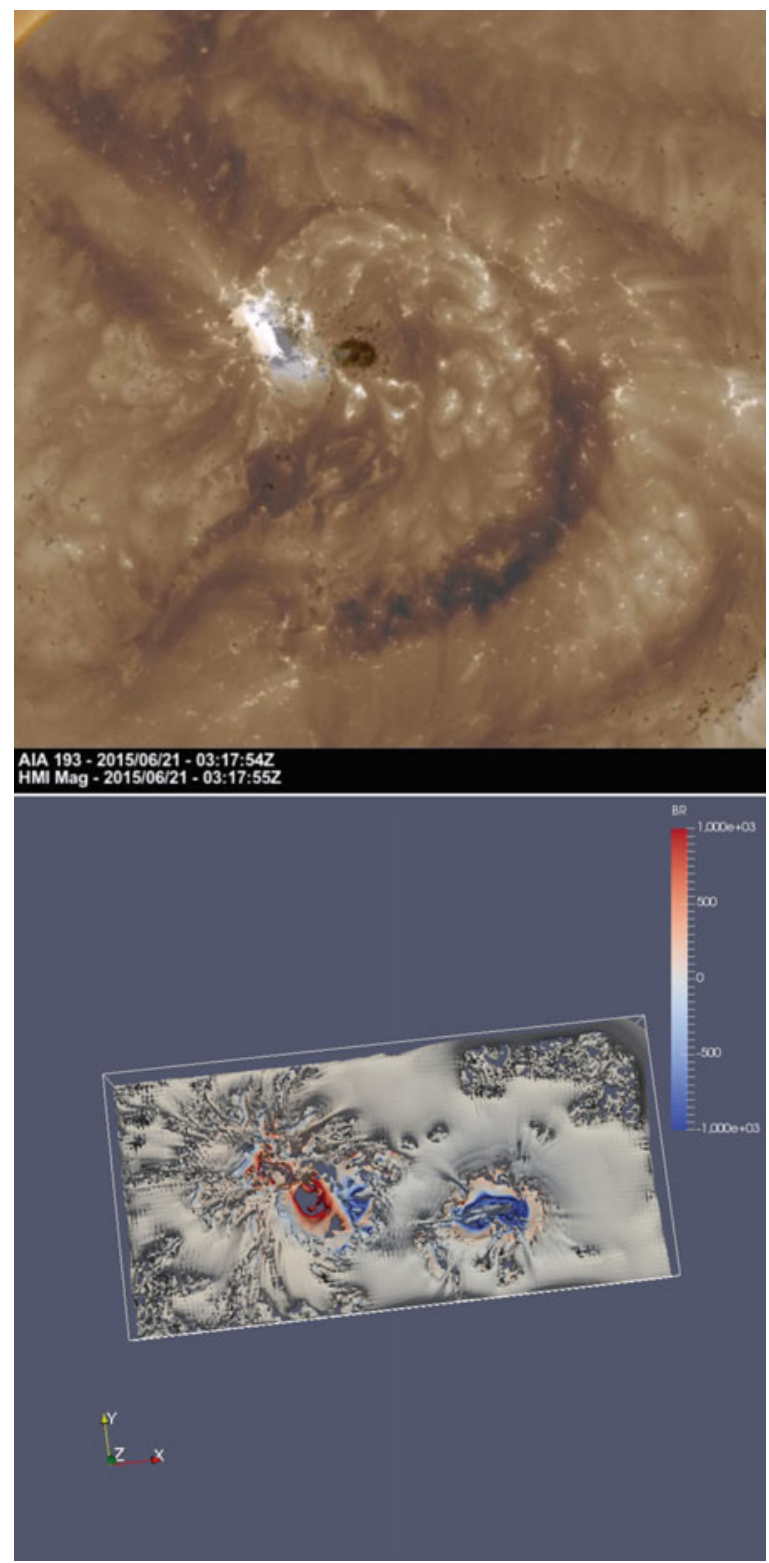

Figure 3. Top: AIA and HMI composition of the AR 12371, made with JHelioviewer. Bottom: decay index computed with a NLFFF extrapolation with SHARPS/HMI data on 21 June 2015.

\section{Conclusions}

The decay index is an indicator of magnetic instability, appropriate mainly for torus instabilities in a particular height and in the area of influence of the torus. However, different approaches may be studied for assessing eruptive instabilities as a whole in a volumetric way, since the magnetic decay with height is a helpful tool. 


\section{Acknowledgements}

We acknowledge the use of publicly available data products from SDO/AIA, SDO/HMI and PFSS and NLFFF Solarsoft packages. We acknowledge and thank JHelioviewer for visualization, and also to Paraview for $3 \mathrm{D}$ plotting.

J. P. acknowledges funding from IAU to attend IAUS327 and UAH-travel grants. She also acknowledges funding from the Spanish MINECO project AYA2013-47735-P.

\section{References}

Amari, T., Luciani, J. F., Aly, J. J., Mikic, Z., \& Linker, J. 2003, ApJ, 585, 1073.

Amari, T., Luciani, J. F., Aly, J. J., Mikic, Z., \& Linker, J. 2003, ApJ, 595, 1231.

Antiochos, S. K., DeVore, C. R., \& Klimchuk, J. A. 1999, ApJ, 510, 485.

Aulanier, G., Török, T., Démoulin, P., \& DeLuca, E. E. 2010, ApJ, 708, 314.

Aulanier, G. 2014, Nature of Prominences and their Role in Space Weather, 300, 184.

Bateman, G. 1978, Cambridge, Mass., MIT Press, 1978. 270.

Cid, C., Palacios, J., Saiz, E., \& Guerrero, A. 2016, ApJ, 828, 11.

Feynman, J. \& Martin, S. F. 1995, JGR, 100, 3355.

Filippov, B. P., Martsenyuk, O. V., Den, O. E., \& Platov, Y. V. 2014, Astronomy Reports, 58, 928.

Forbes, T. G. \& Lin, J. 2000, JASTP, 62, 1499.

Forbes, T. G. \& Priest, E. R. 1995, ApJ, 446, 377.

Hoeksema, J. T., Liu, Y., Hayashi, K., et al. 2014, Solar Phys., 289, 3483.

Kliem, B. \& Török, T. 2006, Physical Review Letters, 96, 255002.

Mackay, D. H., Karpen, J. T., Ballester, J. L., Schmieder, B., \& Aulanier, G. 2010, SSR, 151, 333.

Moore, R. L., Sterling, A. C., Hudson, H. S., \& Lemen, J. R. 2001, ApJ, 552, 833.

Palacios, J., Cid, C., Guerrero, A., Saiz, E., \& Cerrato, Y. 2015, A\& A, 583, A47.

Palacios, J., Cid, C., Guerrero, A., et al. 2016, Coimbra Solar Physics Meeting: Ground-based Solar Observations in the Space Instrumentation Era, 504, 131.

Scherrer, P. H., Schou, J., Bush, R. I., et al., 2012, Solar Phys., 275, 207.

Schrijver, C. J. \& De Rosa, M. L. 2003, Solar Phys., 212, 165.

Török, T. \& Kliem, B. 2005, ApJL, 630, L97.

Török, T. \& Kliem, B. 2007, AN, 328, 743.

van Ballegooijen, A. A. \& Martens, P. C. H. 1989, ApJ, 343, 971.

Wheatland, M. S., Sturrock, P. A., \& Roumeliotis, G. 2000, ApJ, 540, 1150.

Xu, Y., Liu, C., Jing, J., \& Wang, H. 2012, ApJ, 761, 52.

Zuccarello, F. P., Seaton, D. B., Mierla, M., et al. 2014, ApJ, 785, 88.

Zuccarello, F. P., Aulanier, G., \& Gilchrist, S. A. 2015, ApJ, 814, 126.

Zuccarello, F. P., Aulanier, G., \& Gilchrist, S. A. 2016, ApJL, 821, L23. 